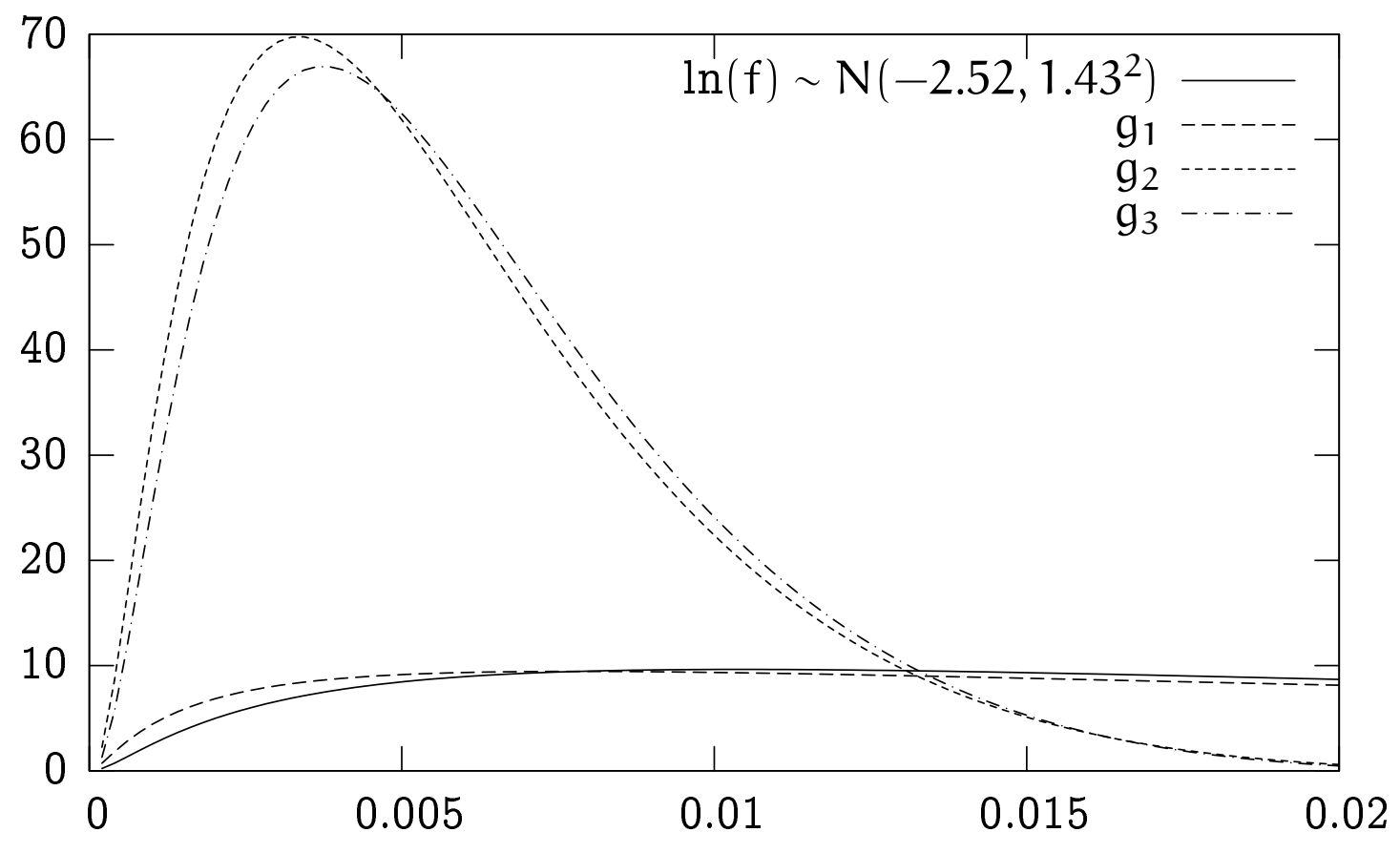

\title{
A practical test for the choice of mixing distribution in discrete choice models
}

\author{
Mogens Fosgerau, Danish Transport Research Inst. \\ Michel Bierlaire, EPFL
}

Conference paper STRC 2006

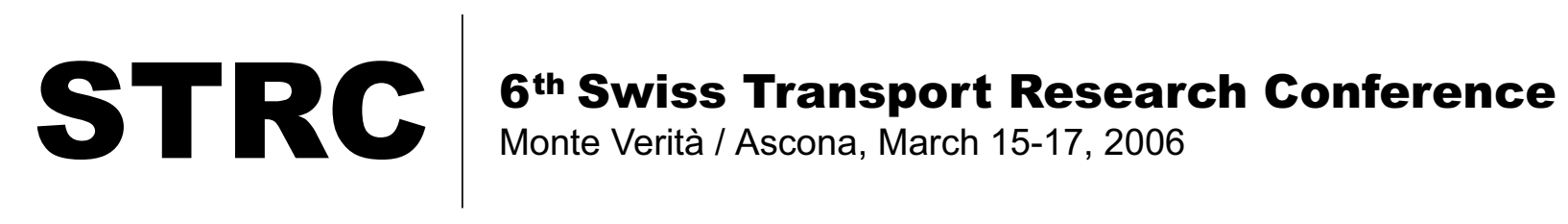




\title{
A practical test for the choice of mixing distribution in discrete choice models
}

\author{
Mogens Fosgerau \\ Danish Transport Research Institute \\ Lyngby
}

Phone: +45 45256521

Fax: +45 45936533

Email:mf@dtf.dk

February 2006

\author{
Michel Bierlaire \\ Institute of Mathematics, EPFL \\ Lausanne \\ Phone: +41216932537 \\ Fax: +41216935570 \\ Email: michel.bierlaire@epfl.ch
}

\begin{abstract}
The choice of a specific distribution for random parameters of discrete choice models is a critical issue in transportation analysis. Indeed, various pieces of research have demonstrated that an inappropriate choice of the distribution may lead to serious biases in model forecast and in the estimated means of random parameters. In this paper, we propose a practical test, based on seminonparametric techniques. The test is analyzed both on synthetic and real data, and is shown to be simple and powerful.
\end{abstract}

\section{Keywords}

Discrete Choice Models - Mixture models - Statistical test - STRC 2006 - Monte Verita 


\section{Introduction}

An important advantage of discrete choice models to analyze transportation demand is their disaggregate nature, allowing them to capture heterogeneity in the population under interest. The analyst identifies segments, typically characterized by socio-economic characteristics such as income, age, or gender, or by the choice context, defined for example by the trip purpose. Each segment must be sampled in order to have a sufficient amount of data to estimate statistically significant models. However, even after controlling for observable characteristics, there is as a rule lots of heterogeneity left. This remaining heterogeneity can be accounted for with random parameters

Except for some specific models (such as probit models with normally distributed coefficients), the error structure of the resulting model becomes very complicated, and cannot be represented by a closed form probability model. We obtain mixtures of models, where the underlying choice model, conditional on the value of the parameters $\beta$ is written as

$$
P_{n}\left(i \mid \mathcal{C}_{n}, \beta\right)
$$

where $\mathcal{C}_{n}$ is the choice set of decision-maker $n, i \in \mathcal{C}_{n}$ is the alternative under consideration and $\beta \in \mathbb{R}^{K}$ is a vector of parameters. Assuming that $\beta$ is randomly distributed with $\operatorname{PDF} f(x)$, the mixture of models is defined by

$$
P_{n}\left(i \mid \mathcal{C}_{n}\right)=\int_{\beta} P_{n}\left(i \mid \mathcal{C}_{n}, \beta\right) f(\beta) d \beta .
$$

In practice, the kernel choice model (1) is often a Multinomial Logit (MNL) model, but any closed form probability model (such as Generalized Extreme Value models) is adequate. Although proposed about 30 years ago (see for instance Electric Power Research Institute, 1977), the use of mixtures of MNL models (MMNL) has only become popular more recently thanks to the increasing power of computers allowing for the systematic use of Monte-Carlo simulation to approximate the complex error structures of these models (see, among many others, discussions by McFadden and Train, 2000, Ben-Akiva et al., 2001, Hensher and Greene, 2003, Train, 2003, Viton, 2004).

This modeling approach has been found to be very useful to capture many complex transportation phenomena, such as the analysis of the value of travel time (e.g. Algers et al., 1998, Hess, Bierlaire and Polak, 2005, Greene et al., 2006) and reliability (e.g. Brownstone and Small, 2005, Small et al., 2005), route choice (see Han et al., 2001, Bekhor et al., 2002, Bierlaire and Frejinger, 2005), airport choice (Hess and Polak, 2005), airline choice (Carrier, 2003), vehicle choice (Brownstone et al., 2000, Hess, Train and Polak, 2005), and congestion pricing (Bhat and Castelar, 2002).

An important issue is the choice of a specific distribution for the random parameters. Actually, various pieces of research have demonstrated that an inappropriate choice of the distribution may lead to serious biases in model forecast and in the estimated mean of random parameters. 
A noticeable example is the Normal distribution, used as a default for many applications. Hess, Bierlaire and Polak (2005) discuss wrong interpretations of willingness-to-pay indicators when normal distributions are considered. Fosgerau (to appear) looks at various distributions and concludes that a bad choice may lead to extreme biases. Hess and Axhausen (2005) have examined how well a wide range of parametric distributions can reproduce given target distributions, which are constructed to reflect common assumptions about taste variation in transport demand models.

We note immediately that using only the goodness-of-fit to compare models does not allow one to reach valid conclusions about the validity of the random parameters distribution. Therefore, we propose a test based on seminonparametric (SNP) techniques to decide if a given distribution is appropriate or not.

The term seminonparametric distinguishes a certain class of models from parametric, nonparametric and semiparametric models. Parametric models are the standard classical models and include, e.g., the MNL or the MMNL models. One specifies a model structure and estimates a number of parameters or deep parameters such as the mean and standard deviation of a model parameter. A nonparametric model has very little structure and is based on local approximations of some kind to the relationship of interest rather than the estimation of parameters. A recent general reference to nonparametric methods is Pagan and Ullah (1999). Examples of nonparametric techniques in a transport context are nonparametric regression (Fosgerau, to appear) and local logit (Fosgerau, 2005a). Semiparametric models are a hybrid between parametric and nonparametric models. They introduce parametric assumptions like the specification of some relationship to be a linear combination of independent variables while perhaps the errors remain nonparametric. A notable semiparametric model for discrete choice data is the Klein and Spady (1993) estimator, which has been applied in the transport context by Horowitz (1993), Fosgerau (2005b) and Fosgerau (to appear). Seminonparametric models are not based on local approximations but use instead series approximations to approximate functions such as densities. SNP methods were introduced by Gallant and Nychka (1987). In this paper, we employ a series approximation to approximate an unknown density and hence our approach is seminonparametric in nature.

In the next section, we describe the general methodology. In Section 3, we illustrate the power of the test on synthetic data, where the "true" distribution is specified and known in advance. We also apply the test on real data in Section 4, where we illustrate instances such that the test rejects the assumed distribution, and instances where it does not. After concluding in Section 5, we provide some technical details for SNP methods based on Legendre polynomials in the Appendix.

\section{Methodology}

We want to test if a random parameter $\omega$ of a discrete choice model follows an a priori postulated distribution. We label this our base distribution with CDF $F$ and density $f$. 
The true distribution may be different from $F$. We denote the true CDF by $G$ and its density by $g$. We can rewrite the distribution $G$ in terms of $F$ as

$$
G(\omega)=Q(F(\omega))
$$

where $Q$ is a monotone function from $[0,1]$ to $[0,1]$. As such, $Q$ is a CDF for a stochastic variable on the unit interval. We can differentiate this to express the density $g$ as

$$
g(\omega)=q(F(\omega)) f(\omega) .
$$

The next step is to approximate $q$ in a seminonparametric fashion. Following Bierens (2005), we let $L_{k}$ be transformed Legendre polynomials (see appendix), which form an orthonormal basis for functions on the unit interval. Defining

$$
q_{N}(x)=1+\sum_{k=1}^{N} \delta_{k} L_{k}(x)
$$

we approximate $q$ by

$$
q(x) \approx \frac{1}{K} q_{N}^{2}(x)
$$

where

$$
K=\int_{-\infty}^{+\infty} q_{N}^{2}(F(\omega)) f(\omega) d \omega
$$

is a normalizing constant such that the density $g$ integrates to 1 . Squaring $q_{N}$ guarantees positivity, so that $g$ is a density. We call the $\delta_{k} L_{k}(x)$ SNP terms and $N$ is the number of such terms.

Bierens (2005) shows that any density on the unit interval can be approximated in this way. This approximation is convenient for several reasons. Legendre polynomials have a recursive definition which is easy to implement in software. Orthonormality of the transformed polynomials is likely to reduce problems with correlation in estimation, and makes it easy to compute the normalizing constant. Indeed, defining $z=F(\omega)$ so that $\omega=F^{-1}(z)$ and $d z=f(\omega) d \omega$, we write (4) as

$$
K=\int_{0}^{1} q_{N}^{2}(z) d z=\int_{0}^{1}\left(1+\sum_{k=1}^{N} \delta_{k} L_{k}(x)\right)^{2} d x=1+\sum_{k=1}^{N} \delta_{k}^{2},
$$

the last equality being obtained from the orthonormality of the polynomials. A great deal of flexibility is obtained to approximate $g$, already with a small number of terms, and flexibility can be gradually increased by adding more terms, if necessary.

Assume now that $\beta$ is a parameter of a discrete choice model. The probability for alternative $i$ to be chosen in choice set $\mathcal{C}$ is given by

$$
P_{n}\left(i \mid \mathcal{C}_{n}\right)=\int_{-\infty}^{+\infty} P_{n}\left(i \mid \beta, \mathcal{C}_{n}\right) g(\beta) d \beta
$$


where $P_{n}\left(i \mid \beta, \mathcal{C}_{n}\right)$ is a closed form model, such as the Generalized Extreme Value model (McFadden, 1978). Then,

$$
\begin{aligned}
P_{n}\left(i \mid \mathcal{C}_{n}\right) & \approx \frac{1}{K} \int_{-\infty}^{+\infty} P_{n}\left(i \mid \beta, \mathcal{C}_{n}\right) q_{N}^{2}(F(\beta)) f(\beta) d \beta \\
& =\frac{1}{K} \int_{0}^{1} P_{n}\left(i \mid F^{-1}(z), \mathcal{C}_{n}\right) q_{N}^{2}(z) d z
\end{aligned}
$$

where, again, $z=F(\beta)$. This integral is approximated by Monte-Carlo simulation, and the term $F^{-1}(z)$ corresponds to the draws of the base distribution.

Now, under the null hypothesis that the base distribution is the true distribution, we have $f=g$, which implies that $q$ is identically 1 and thus that $\delta_{k}=0$, for all $k$ in (3). Then the model

$$
P_{n}\left(i \mid \mathcal{C}_{n}\right)=\int_{-\infty}^{+\infty} P_{n}\left(i \mid \beta, \mathcal{C}_{n}\right) g(\beta) d \beta,
$$

is equivalent to the model

$$
P_{n}\left(i \mid \mathcal{C}_{n}\right)=\int_{-\infty}^{+\infty} P_{n}\left(i \mid \beta, \mathcal{C}_{n}\right) f(\beta) d \beta .
$$

By construction, model (6) is a special case of model (5) where all coefficients (except the constant) of the polynomial approximation of $q$ are set to 0 . Consequently, a likelihood ratio test for nested hypotheses is appropriate to test the null hypothesis. If $\mathcal{L}_{U}$ is the loglikelihood of the sample with model (5), and $\mathcal{L}_{R}$ is the loglikelihood of the sample with model (6), then, under $H_{0}: f=g$, the likelihood ratio statistic

$$
-2\left(\mathcal{L}_{R}-\mathcal{L}_{U}\right)
$$

is $\chi^{2}$ distributed with $N$ degrees of freedom, where $N$ is the number of terms considered in the polynomial approximation.

Note that the number of SNP terms must be chosen in advance. Increasing the number of SNP terms makes the alternative hypothesis more general but also increases the demand on the data. Our experience reported later in this paper suggests that 2 or 3 SNP terms give a large degree of flexibility, which may be sufficient for most purposes, while 1 SNP term is not always sufficient to reject a false null hypothesis.

\section{Simulation study}

We first illustrate the concept on semi-simulated data, in order to measure the power (that is, the ability to reject false hypotheses) and the size (that is, the rate at which true hypotheses are rejected) of the test. By semi-simulated data, we mean that we have used an existing database, and performed sample enumeration with a prespecified "true" model to generate simulated choices. 
The data derive from a stated choice experiment, which is part of the Danish value of time study. Some design considerations for this study are detailed in Burge et al. (2004). We have selected a route choice experiment concerning a recent trip by bus. The experimental design is particularly simple involving only in-vehicle travel time and cost. By design, the ratio of cost to time differences range between 1 and 200 DKK per hour (1 EUR $\approx 7.5 \mathrm{DKK}$ ).

Each respondent made 9 choices, one of which was always a dominated choice included as a check on respondents. Respondents who failed to choose the dominant alternative were excluded from the analysis. So were all dominant choices ${ }^{1}$. This leaves 1070 respondents who carried out an average of 7.7 non-dominated choices each.

The true model is specified as a binary model based on the following utility function:

$$
U_{j n t}=\beta_{T n} \mathrm{TT}_{j n t}+\beta_{C} \mathrm{TC}_{j n t}+\varepsilon_{j n t}
$$

where $U_{j n t}$ is the utility associated with alternative $j$ by individual $n$ for question number $t$, $\beta_{T n}$ is a random coefficient distributed across individuals, $\beta_{C}$ is fixed and $\varepsilon_{j n t}$ are i.i.d. extreme value distributions, so that

$$
P_{n}\left(i \mid \mathcal{C}_{n}=\{i, j\}, \beta_{T n}\right)=\prod_{t} P_{n t}\left(i \mid \mathcal{C}_{n}, \beta_{T n}\right)
$$

and

$$
P_{n t}\left(i \mid \mathcal{C}_{n}, \beta_{T n}\right)=\operatorname{Pr}\left(U_{i n t} \geq U_{j n t}\right)=\frac{e^{\beta_{T n} \mathrm{TT}_{i n t}+\beta_{C} \mathrm{TC}_{i n t}}}{\sum_{k=i, j} e^{\beta_{T n} \mathrm{TT}_{k n t}+\beta_{C} \mathrm{TC}_{k n t}}} .
$$

The simulated choices were generated using a cost coefficient of -0.3 and time coefficients following either a normal or a lognormal distribution. These two distributions were chosen to have the same mean and variance and most of their mass within the range of time-cost trade-offs in the data. More specifically, with cost in DKK and time in minutes, the normal distribution had mean 0.5 and standard deviation 0.2 , while the lognormal distribution had mean -1 and standard deviation 0.6 in the underlying normal distribution.

A total of 100 data sets have been generated for each "true" model. We have applied the test using one SNP term. We have tested two null hypotheses: (i) the true distribution is normal and (ii) the true distribution is lognormal. The estimations have been performed ${ }^{2}$ with simulated maximum likelihood, using a total of 500 Halton draws. The number of rejected models is reported in Table 1. At the $95 \%$ level of confidence, the null hypothesis that the true distribution of $\beta_{T}$ is normal is (falsely) rejected $9 \%$ of the times with the first model, and (correctly) rejected $100 \%$ of the times with the second model. The null hypothesis that the true distribution of $\beta_{T}$ is lognormal is (correctly) rejected $99 \%$ of the times with the first model, and (falsely) rejected $5 \%$ of the times with the second model.

\footnotetext{
${ }^{1}$ Due to rounding, there could be other choices that did not involve a positive price of time. They are similarly treated as dominant and removed from the sample.

${ }^{2}$ All estimations are carried out in Ox (Doornik, 2001). The code is available from the authors upon request.
} 


\begin{tabular}{llrrr} 
& & & \multicolumn{2}{c}{$H_{0}$} \\
\cline { 3 - 5 } & & & Normal & Lognormal \\
\hline $95 \%$ & True dist: & Normal & 9 & 99 \\
& True dist: & Lognormal & 100 & 5 \\
\hline $99 \%$ & True dist: & Normal & 1 & 78 \\
& True dist: & Lognormal & 88 & 0 \\
\hline
\end{tabular}

Table 1: Simulated data: number of rejections with 1 SNP term

We analyze these results in more details in Figures 1 and 2, where the cumulative distribution of the likelihood ratio statistic is reported for the $4 \times 100$ experiments. Figure 1 reports the results for testing the null hypothesis that the true distribution is a normal (corresponding to the first column in Table 1), and Figure 2 reports the results for testing the null hypothesis that the true distribution is a lognormal. The threshold for the $95 \%$ test is shown $(3.84$, from the $\chi^{2}$ distribution with one degree of freedom), as well as the $99 \%$ (6.63). At the $99 \%$ level of confidence, the number of false rejections drops, as well as the number of correct rejections, as reported in Table 1.

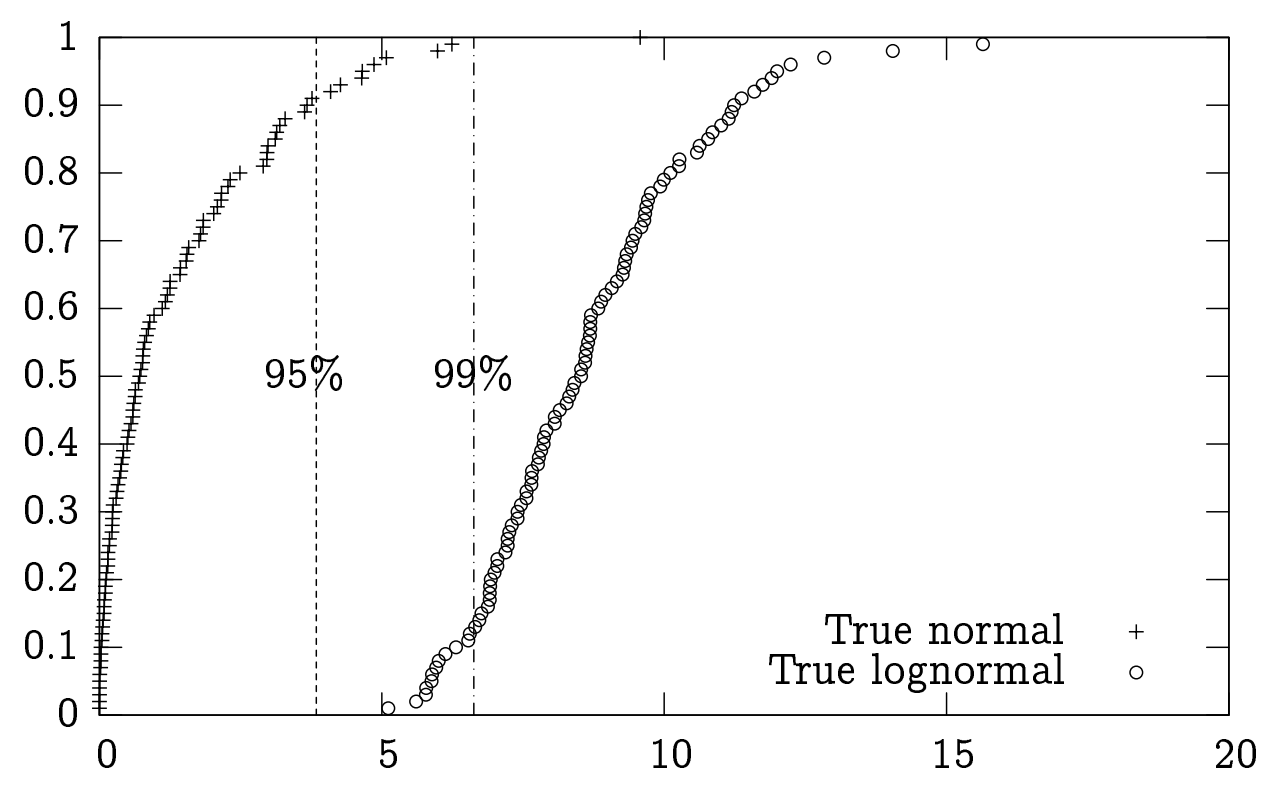

Figure 1: Distribution of the likelihood ratio for 100 experiments under $H_{0}=$ "true normal"

Although both the power and the size of the test are very good when just one SNP term is used, we have also applied the test with two SNP terms. The results are reported in Table 2.

The test seems to perform very well in these circumstances. The power of the test is very high, allowing us to reject a very large proportion of false nulls, even at the $99 \%$ level of confidence. 


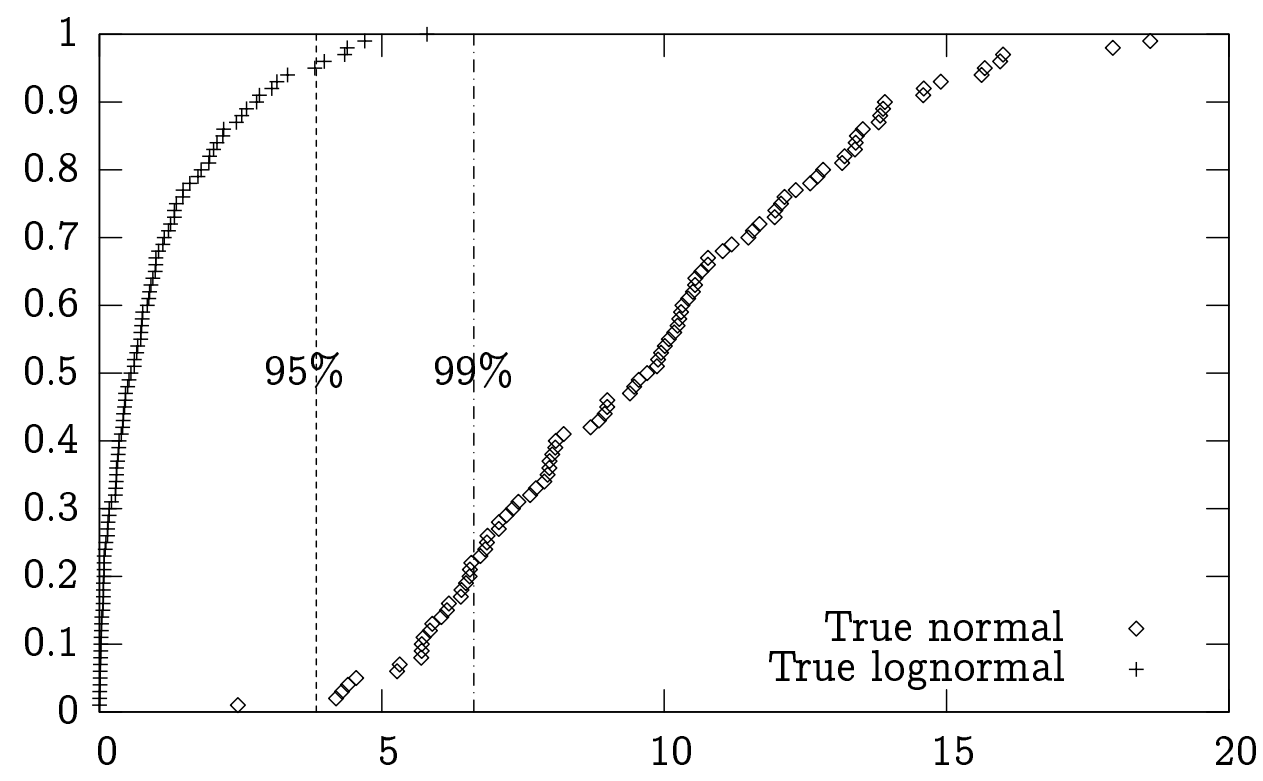

Figure 2: Distribution of the likelihood ratio for 100 experiments under $H_{0}=$ "true lognormal"

\begin{tabular}{lrrrr} 
& & \multicolumn{2}{c}{$H_{0}$} \\
\cline { 3 - 5 } & & & Normal & Lognormal \\
\hline $95 \%$ & True dist: & Normal & 9 & 100 \\
& True dist: & Lognormal & 100 & 3 \\
\hline $99 \%$ & True dist: & Normal & 4 & 99 \\
& True dist: & Lognormal & 100 & 0 \\
\hline
\end{tabular}

Table 2: Simulated data: number of rejections with 2 SNP terms

The actual size seems to differ from the nominal size in both directions. Consequently, it may be appropriate to increase the critical value in practice.

\section{Case study}

We now apply the test to the real data set, using again the model specification (7). We test the model with one SNP term, where the base distribution of $\beta_{T}$ is a normal. The results of the two estimations are reported in Table 3. The likelihood ratio (LR) test is

$$
-2(-4153.57+4150.14)=6.86
$$

and the $H_{0}$ hypothesis that $\beta_{T}$ follows a normal distribution can be rejected at the $99 \%$ level of confidence. Note that the coefficient $\delta_{1}$ of the SNP term is significantly different from 0 . A 
visual comparison of the estimated densities of the normal in the first model and of $g$ in the second shows however that they look quite similar (see Figure 3). Moreover, the estimated VTTS are quite similar. This shows that informal tests may be misleading in this context, and that a formal likelihood ratio test is necessary.

\begin{tabular}{|c|c|c|c|c|c|c|}
\hline \multirow{2}{*}{$\mathcal{L}=$} & \multicolumn{3}{|c|}{$\beta_{T} \sim f(x)=N\left(\mu, \sigma^{2}\right)$} & \multicolumn{3}{|c|}{$\beta_{T} \sim g(x)$} \\
\hline & Estim. & Std.err. & $t$-test & Estim. & Std.err. & $t$-value \\
\hline$\beta_{C}$ & -0.36 & 0.01 & -25.1 & -0.35 & 0.01 & -25.3 \\
\hline$\mu\left(\beta_{T}\right)$ & 0.03 & 0.01 & 1.9 & -0.15 & 0.06 & -2.7 \\
\hline$\sigma\left(\beta_{T}\right)$ & 0.34 & 0.01 & 24.4 & 0.38 & 0.02 & 16.2 \\
\hline & & & & 0.25 & 0.08 & 3.3 \\
\hline VTTS $(\mathrm{DKK} / \mathrm{h})$ & 25.33 & & & 25.49 & & \\
\hline
\end{tabular}

Table 3: Testing a normal distribution

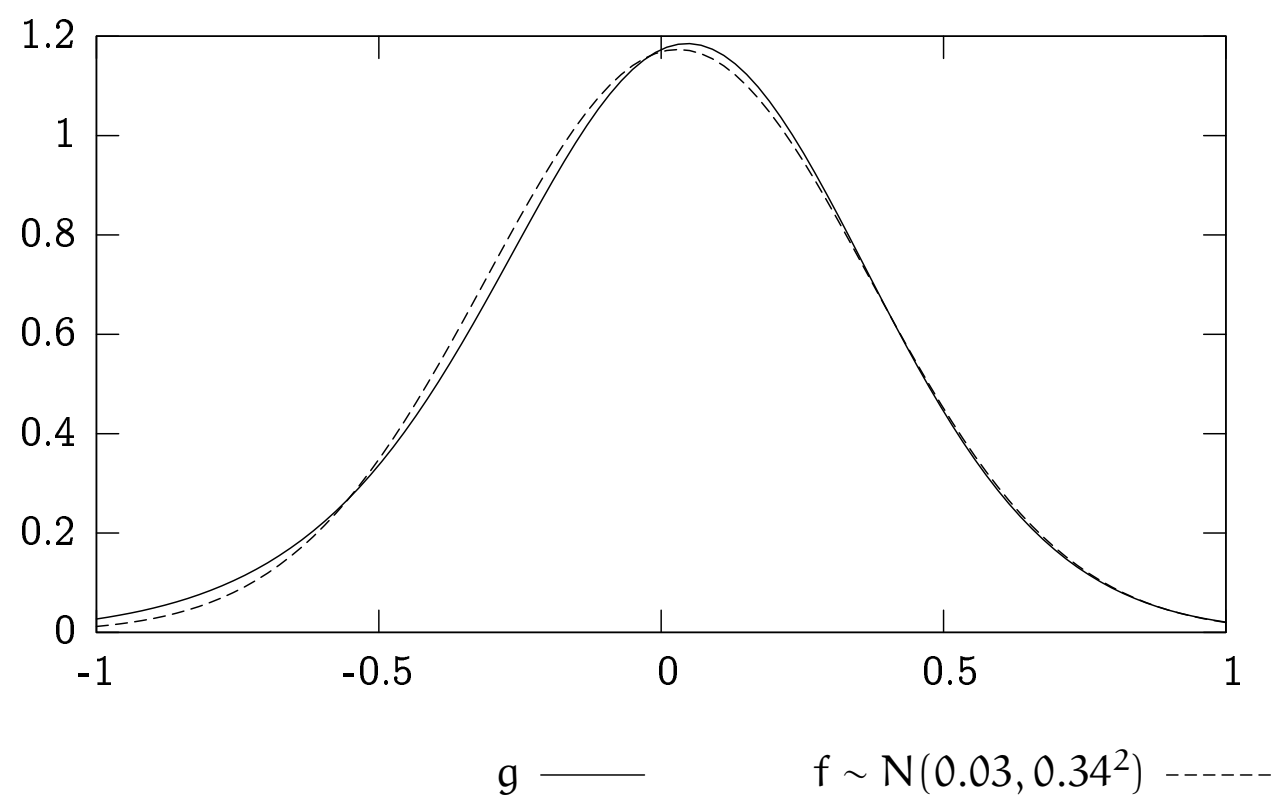

Figure 3: Comparison of $f$ and $g, f$ normal

We now test the model with one SNP term, where the base distribution of $\beta_{T}$ is lognormal. The results of the two estimations are reported in Table 4.

The LR test is

$$
-2(-4304.32+4302.94)=2.76
$$

and the $H_{0}$ hypothesis that $\beta_{T}$ follows a lognormal distribution cannot be rejected at the $95 \%$ level of confidence and not even at the $85 \%$ level. A visual comparison of the densities of the 
lognormal in the first model and of $g$ in the second show no visible differences (see Figures 4 and 5), and the VTTS is about the same with the two models. However, the $\delta_{1}$ parameter of the Legendre polynomial appears to be significantly different from zero, which indicates that the case could be analyzed in more details.

\begin{tabular}{rrrr|rrr}
\multirow{2}{*}{$\ln \left(\beta_{T}\right) \sim f(x)=N\left(\mu, \sigma^{2}\right)$} & \multicolumn{3}{c}{$\beta_{T} \sim g(x)$} \\
\cline { 2 - 7 } & \multicolumn{3}{c}{-4304.32} & \multicolumn{3}{c}{-4302.94} \\
\cline { 2 - 7 }$\mu$ Estim. & Std.err. & $t$-test & Estim. & Std.err. & $t$-value \\
\hline$\beta_{C}$ & -0.45 & 0.01 & -38.3 & -0.45 & 0.02 & -28.7 \\
$\sigma\left(\beta_{T}\right)$ & -2.52 & 0.05 & -46.8 & -2.92 & 0.06 & -46.5 \\
$\left.\beta_{T}\right)$ & 1.43 & 0.05 & 30.6 & 1.50 & 0.04 & 36.8 \\
$\delta_{1}$ & & & & 0.14 & 0.04 & 3.3 \\
VTTS (DKK/h) & 30.48 & & & 32.13 & &
\end{tabular}

Table 4: Testing a lognormal distribution

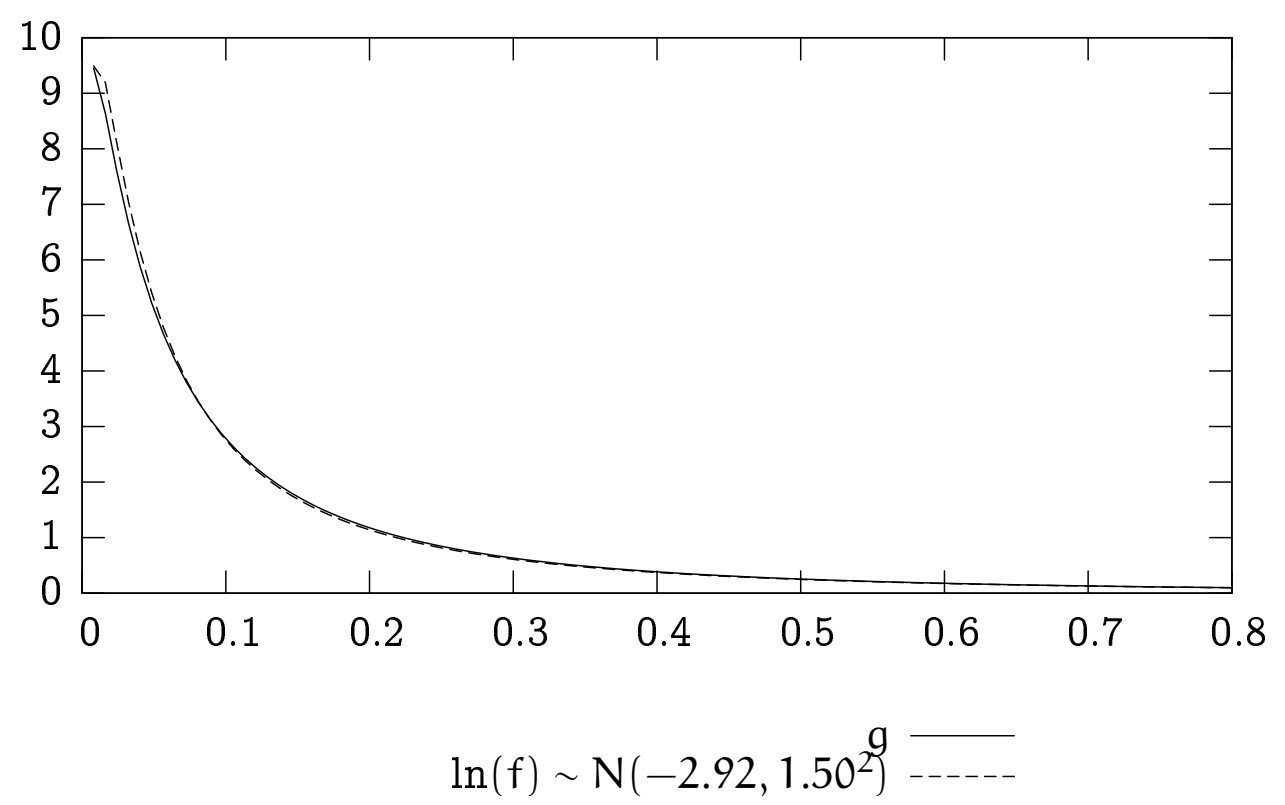

Figure 4: Comparison of $f$ and $g, f$ lognormal

Consequently, we have performed the test with 2 and 3 SNP terms. The results are presented in Table 5, where we denote by $g_{N}$ the distribution obtained with $N$ SNP terms. We obtain a large improvement in the likelihood when a second SNP term is added. Also, the average VTTS significantly changes between the model with one and the model with two terms. The likelihood ratio test for the model with two terms in the polynomial is

$$
-2(-4304.32+4263.57)=81.50
$$




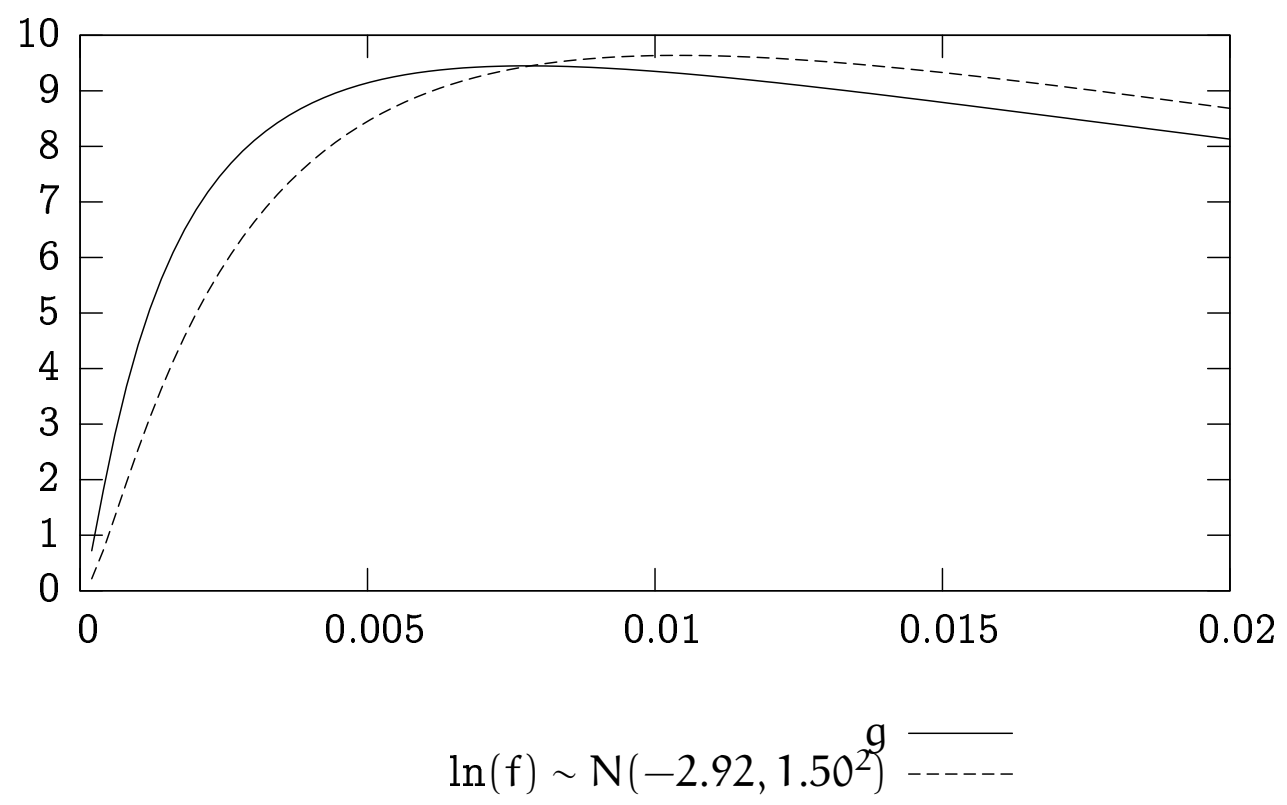

Figure 5: Comparison of $f$ and $g, f$ lognormal (zoom)

which is far beyond the 5.99 threshold of the $95 \%$ level, and even far beyond the 9.21 threshold of the $99 \%$ level. Therefore, we clearly reject the lognormal in this case. A visual comparison (Figures 6 and 7) illustrates well the cause of this rejection.

As a final note on this example, we emphasize the fact that the likelihood with the normal distribution is much better than with the lognormal, even though the normal distribution is also rejected.

This suggests that the model might be misspecified. Therefore, we now estimate a different model specification on the same data set. The idea is to specify a model in the VTTS space, as suggested by Train and Weeks (2005). This model, proposed by Fosgerau (to appear), is defined as

$$
y_{n t}=1\left(\beta_{n}<\ln \frac{C_{2 n t}-C_{1 n t}}{T_{1 n t}-T_{2 n t}}+\eta_{n t}\right) .
$$

It can be written as a classical binary random utility model, with

$$
\begin{aligned}
& U_{1 n t}=\lambda \ln \frac{C_{2 n t}-C_{1 n t}}{T_{1 n t}-T_{2 n t}}+\varepsilon_{1 n t} \\
& U_{2 n t}=\lambda \beta_{n}+\varepsilon_{2 n t}
\end{aligned}
$$

where it is assumed without loss of generality that the alternatives are numbered such that $T_{1 n t}>T_{2 n t}$ for each observation. Also, we assume that there is no dominated alternative, that is $C_{1 n t}<C_{2 n t}$, for all observations, so that the argument of the logarithm is always positive. The unknown parameter $\beta_{n}$ is interpreted as the logarithm of the Value of Travel Time Savings of individual $n$, and $\lambda$ is a scale parameter. 


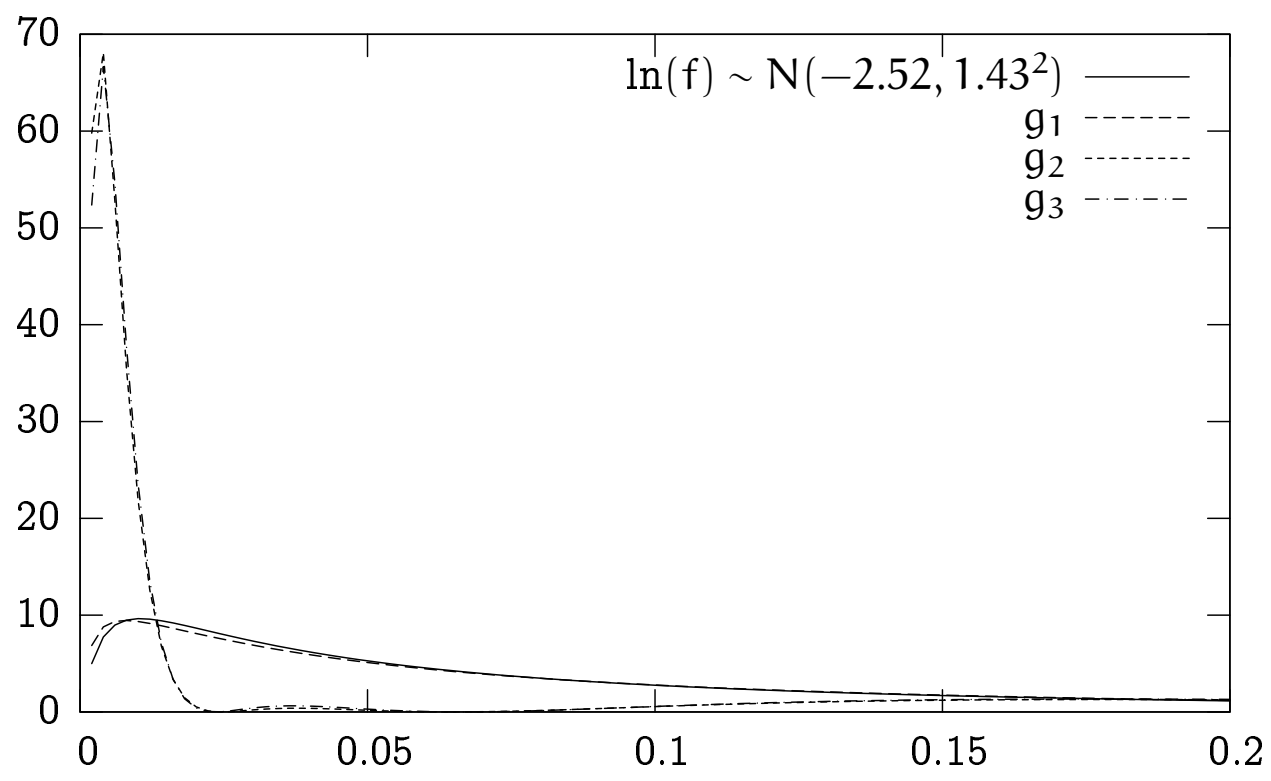

Figure 6: Comparison of $f, g_{1}, g_{2}$ and $g_{3}, f$ lognormal

We test the model with one, two and three SNP terms, where the base distribution for $\beta_{n}$ is normal. The results are reported in Table 6 . Note that the values of the likelihood obtained with this new specification are much better than those obtained previously.

Looking at the model with one SNP term, the likelihood ratio statistic is

$$
-2(-3732.04+3730.01)=4.06
$$

which is just above the 3.84 threshold of the $95 \%$, proposing a weak rejection. Inflating slightly the level to $96 \%$ gives a threshold of 4.22 which is not reached. From the conclusions of the simulation study in Section 3, we decide not to reject the normal based on this test. We reach the same conclusion with the model with 2 SNP terms, where the test statistic is

$$
-2(-3732.04+3728.83)=6.42
$$

which is just above the 5.99 threshold of the $95 \%$ level, but does not reach the 6.44 threshold of the $96 \%$ level. Finally, the test with three SNP terms give

$$
-2(-3732.04+3727.79)=8.50
$$

which is just above the 7.81 threshold of the $95 \%$ level, and under the 8.95 threshold of the $97 \%$ level. Therefore, even with more terms, the test suggests not to reject the normal distribution in this model. 


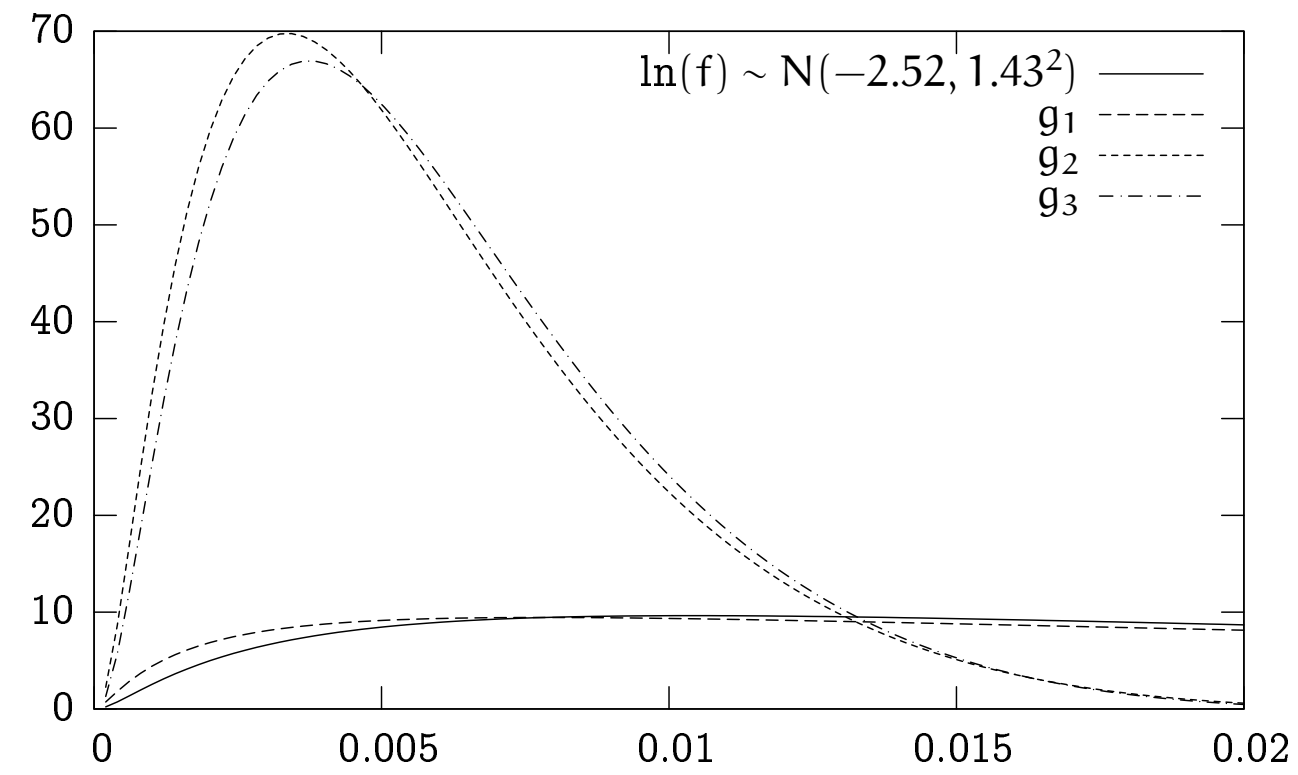

Figure 7: Comparison of $f, g_{1}, g_{2}$ and $g_{3}, f$ lognormal (zoom)

\section{Conclusion}

We have proposed a method based on a seminonparametric (SNP) specification to test if a random parameter of a discrete choice model indeed follows a given distribution.

The simulation study shows that the test is well able to discriminate between normal and lognormal with $1 \mathrm{SNP}$ term only. It also suggests that it is good practice to look for a slightly higher loglikelihood difference before rejecting a distribution.

We have demonstrated the application of the test on two case studies. In preference space, we reject the normal with 1 SNP term. The lognormal is not rejected with 1 term but it is clearly rejected with two terms. So we conclude that it is generally desirable to include two or three SNP terms in order to test the base distribution against an alternative that is as general as possible. There are relevant alternatives which are not captured with just $1 \mathrm{SNP}$ term. However, there seems to be little point in using more than 2 or 3 SNP terms.

The first case study showed that the significant improvement in the likelihood with the model with a normal distribution is not sufficient to accept the distribution, which was actually rejected by the test. It was rather evidence of model misspecification, which motivates the model in VTTS space analyzed in the second case study, as suggested by Fosgerau (to appear).

In this case, the likelihood is dramatically improved relative to all previous models. For each test with 1, 2 and 3 SNP terms, we get a weak rejection of the normal using the nominal test level of $95 \%$. If we inflate this level slightly as suggested by the simulation study, we conclude that the normal distribution cannot be rejected for $\beta$, the logarithm of the VTTS. 
In summary, the test works well on simulated data, and we have shown a successful application of the test to real data. It can reject bad models and, by including more terms for flexibility, show how a particular distribution may fail. So we believe that it is a very powerful tool for concrete applications.

In the future, it would be interesting to adapt the test for more than one random parameter. A simple heuristic would consist in testing each parameter independently. The robustness of this approach must be analyzed both in the case of independent and correlated random parameters. Also, the practical use of multivariate polynomial approximations should be assessed.

Note that other uses of the model are possible. Namely, one can treat the SNP extension to some base distribution as a way of generating a larger model universe with greater flexibility allowed for the mixing distribution. As the sample size and the number of SNP terms tends to infinity, one could hope that the model would be capable of approximating any true mixing distribution. Identification and consistency of this procedure is the subject of ongoing research. Note however that the trade off between model flexibility and data overfitting must always be considered by the analyst.

\section{A Appendix: Legendre polynomials}

The Legendre polynomials $\widehat{L}_{n}(x)$ are defined by

$$
\widehat{L}_{n}(x)=\sum_{m=0}^{M}(-1)^{m} \frac{(2 n-2 m) !}{2^{n} m !(n-m) !(n-2 m) !} x^{n-2 m}
$$

where $M=n / 2$ or $M=(n-1) / 2$, whichever is an integer. They can also be defined recursively,

$$
\widehat{L}_{n}(x)=\left((2 n-1) x \widehat{L}_{n-1}(x)-(n-1) \widehat{L}_{n-2}(x)\right) / n,
$$

where $\widehat{L}_{0}(x)=1$ and $\widehat{L}_{1}(x)=x$ (see Abramowitz and Stegun, 1972, chap. 8 and 22). They are orthogonal on $[-1,1]$ in the sense that

$$
\int_{-1}^{1} \widehat{L}_{m}(x) \widehat{L}_{n}(x) d x=0 \text { if } m \neq n .
$$

In our context, it is more appropriate for the polynomials to be orthogonal on $[0,1]$, as the arguments are defined by a CDF. Therefore, Bierens (2005) proposes the following transformation:

$$
L_{n}(x)=\sqrt{2 n+1} \widehat{L}_{n}(2 x-1)
$$

so that they are orthonormal on $[0,1]$, that is

$$
\int_{0}^{1} L_{m}(x) L_{n}(x) d x= \begin{cases}0 & \text { if } m \neq n \\ 1 & \text { if } m=n .\end{cases}
$$


The recursive definition of these transformed polynomials is given by

$$
L_{n}(x)=\frac{\sqrt{4 n^{2}-1}}{n}(2 x-1) L_{n-1}(x)-\frac{(n-1) \sqrt{2 n+1}}{n \sqrt{2 n-3}} L_{n-2}(x) .
$$

The first polynomials are

$$
\begin{aligned}
& L_{0}(x)=1 \\
& L_{1}(x)=\sqrt{3}(2 x-1) \\
& L_{2}(x)=\sqrt{5}\left(6 x^{2}-6 x+1\right) \\
& L_{3}(x)=\sqrt{7}\left(20 x^{3}-30 x^{2}+12 x-1\right) .
\end{aligned}
$$

\section{References}

Abramowitz, M. and Stegun, I. A. (eds) (1972). Handbook of Mathematical Functions with Formulas, Graphs, and Mathematical Tables, 9th edn, Dover, New York.

Algers, S., Bergstrom, P., Dahlberg, M. and Dillen, J. (1998). Mixed logit estimation of the value of travel time, Technical Report 1998:15, Uppsala - working paper series. available at http://ideas.repec.org/p/fth/uppaal/199815.html.

Bekhor, S., Ben-Akiva, M. and Ramming, M. S. (2002). Adaptation of logit kernel to route choice situations, Transportation Research Record 1805: 78-85.

Ben-Akiva, M., Bolduc, D. and Walker, J. (2001). Specification, identification, and estimation of the logit kernel (or continuous mixed logit) model, Technical report, Department of Civil Engineering, MIT, Cambridge, Ma.

Bhat, C. R. and Castelar, S. (2002). A unified mixed logit framework for modeling revealed and stated preferences: formulation and application to congestion pricing analysis in the San Francisco Bay area, Transportation Research 36B: 593-616.

Bierens, H. J. (2005). Semi-nonparametric modeling of densities on the unit interval, with applications to censored mixed proportional hazard models and ordered probability models: Identification and consistency results, Econometric Society World Congress.

Bierlaire, M. and Frejinger, E. (2005). Route choice models with subpath components, Proceedings of the 5th Swiss Transportation Research Conference. www.strc.ch.

Brownstone, D., Bunch, D. and Train, K. (2000). Joint mixed logit models of stated and revealed preferences for alternative-fuel vehicles, Transportation Research Part B 34: 315-338.

Brownstone, D. and Small, K. (2005). Valuing time and reliability: assessing the evidence from road pricing demonstrations, Transportation Research Part A 39(4): 279-293. 
Burge, P., Rohr, C., Vuk, G. and Bates, J. (2004). Review of international experience in VOT study design, Proceedings of the European Transport Conference.

Carrier, E. (2003). Modeling Airline Passenger Choice: Passenger Preference for Schedule in the Passenger Origin-Destination Simulator (PODS), $\mathrm{PhD}$ thesis, Massachusetts Institute of Technology.

Doornik, J. A. (2001). Ox: An Object-Oriented Matrix Language, Timberlake Consultants Press, London.

Electric Power Research Institute (1977). Methodology for predicting the demand for new electricity-using goods, Final report. Project 488-1 EA-593, Electric Power Research Institute, Palo Alto, Ca.

Fosgerau, M. (2005a). Specification of a model to measure the value of travel time savings, European Transport Conference.

Fosgerau, M. (2005b). Unit income elasticity of the value of travel time savings, European Transport Conference.

Fosgerau, M. (to appear). Investigating the distribution of the value of travel time savings, Transportation Research Part B .

Gallant, A. R. and Nychka, D. W. (1987). Semi-nonparametric maximum likelihood estimation, Econometrica 55(2): 363-390.

Greene, W. H., Hensher, D. A. and Rose, J. (2006). Accounting for heterogeneity in the variance of unobserved effects in mixed logit models, Transportation Research Part B 40(1): 7592.

Han, B., Algers, S. and Engelson, L. (2001). Accommodating drivers' taste variation and repeated choice correlation in route choice modeling by using the mixed logit model, 80th Annual Meeting of the Transportation Research Board.

Hensher, D. and Greene, W. (2003). The mixed logit model: the state of practice, Transportation 30(2): 133-176.

Hess, S. and Axhausen, K. W. (2005). Distributional assumptions in the representation of random taste heterogeneity, Proceedings of the 5th Swiss Transportation Research Conference. www.strc.ch.

Hess, S., Bierlaire, M. and Polak, J. (2005). Estimation of value of travel-time savings using mixed logit models, Transportation Research Part A 39(3): 221-236. 
Hess, S. and Polak, J. (2005). Mixed logit modelling of airport choice in multi-airport regions, Journal of Air Transport Management 11(2): 59-68.

Hess, S., Train, K. and Polak, J. (2005). On the use of modified latin hypercube sampling (MLHS) method in the estimation of mixed logit model for vehicle choice, Transportation Research Part A . Forthcoming, accepted for publication.

Horowitz, J. (1993). Semiparametric estimation of a work trip mode choice model, Journal of Econometrics 58: 49-70.

Klein, R. and Spady, R. (1993). An efficient semiparametric estimator for binary response models, Econometrica 61(2): 387-422.

McFadden, D. (1978). Modelling the choice of residential location, in A. Karlquist et al. (ed.), Spatial interaction theory and residential location, North-Holland, Amsterdam, pp. 7596.

McFadden, D. and Train, K. (2000). Mixed MNL models for discrete response, Journal of Applied Econometrics 15(5): 447-470.

Pagan, A. and Ullah, A. (1999). Nonparametric Econometrics, Cambridge University Press.

Small, K. A., Winston, C. and Yan, J. (2005). Uncovering the distribution of motorists' preferences for travel time and reliability, Econometrica 73(4): 1367-1382.

Train, K. (2003). Discrete Choice Methods with Simulation, Cambridge University Press. http://emlab.berkeley.edu/books/choice.html.

Train, K. and Weeks, M. (2005). Discrete choice models in preference space and willingnessto-pay space, in R. Scarpa and A. Alberini (eds), Applications of simulation methods in environmental and resource economics, The Economics of Non-Market Goods and Resources, Springer, pp. 1-16.

Viton, P. A. (2004). Will mixed logit change urban transport policies?, Journal of Transport Economics and Policy 38(3): 403-423. 


\begin{tabular}{|c|c|c|c|c|c|c|c|c|c|c|c|c|}
\hline \multirow[t]{2}{*}{$\mathcal{L}=$} & \multicolumn{3}{|c|}{$\ln \left(\beta_{T}\right) \sim f(x)=N\left(\mu, \sigma^{2}\right)$} & \multicolumn{3}{|c|}{$\begin{array}{c}\beta_{T} \sim g_{1}(x) \\
-4302.94\end{array}$} & \multicolumn{3}{|c|}{$\begin{array}{c}\beta_{T} \sim g_{2}(x) \\
-4263.57\end{array}$} & \multicolumn{3}{|c|}{$\begin{array}{c}\beta_{T} \sim g_{3}(x) \\
-4263.29\end{array}$} \\
\hline & Estim. & Std.err. & t-test & Estim. & Std.err. & t-test & Estim. & Std.err. & t-test & Estim. & Std.err. & t-test \\
\hline$\beta_{C}$ & -0.45 & 0.01 & -38.3 & -0.45 & 0.02 & -28.7 & -0.45 & 0.01 & -38.5 & -0.45 & 0.01 & -34.94 \\
\hline$\mu\left(\beta_{T}\right)$ & -2.52 & 0.05 & -46.8 & -2.92 & 0.06 & -46.5 & -3.25 & 0.04 & -86.9 & -3.16 & 0.03 & -92.28 \\
\hline$\sigma\left(\beta_{T}\right)$ & 1.43 & 0.05 & 30.6 & 1.50 & 0.04 & 36.8 & 1.29 & 0.02 & 51.7 & 1.26 & 0.02 & 63.80 \\
\hline$\delta_{1}$ & & & & 0.14 & 0.04 & 3.3 & -0.07 & 0.05 & -1.52 & -0.02 & 0.08 & -0.19 \\
\hline$\delta_{2}$ & & & & & & & 1.20 & 0.25 & 4.76 & 1.29 & 0.32 & 4.07 \\
\hline$\delta_{3}$ & & & & & & & & & & -0.12 & 0.13 & -0.92 \\
\hline VTTS (DKK/h) & 30.48 & & & 32.13 & & & 52.85 & & & 46.56 & & \\
\hline LR against 0 & & & & 2.76 & & & 81.50 & & & 82.06 & & \\
\hline LR against 1 & & & & & & & 78.74 & & & 79.30 & & \\
\hline LR against 2 & & & & & & & & & & 0.56 & & \\
\hline
\end{tabular}

Table 5: Testing a lognormal distribution with more than one term 


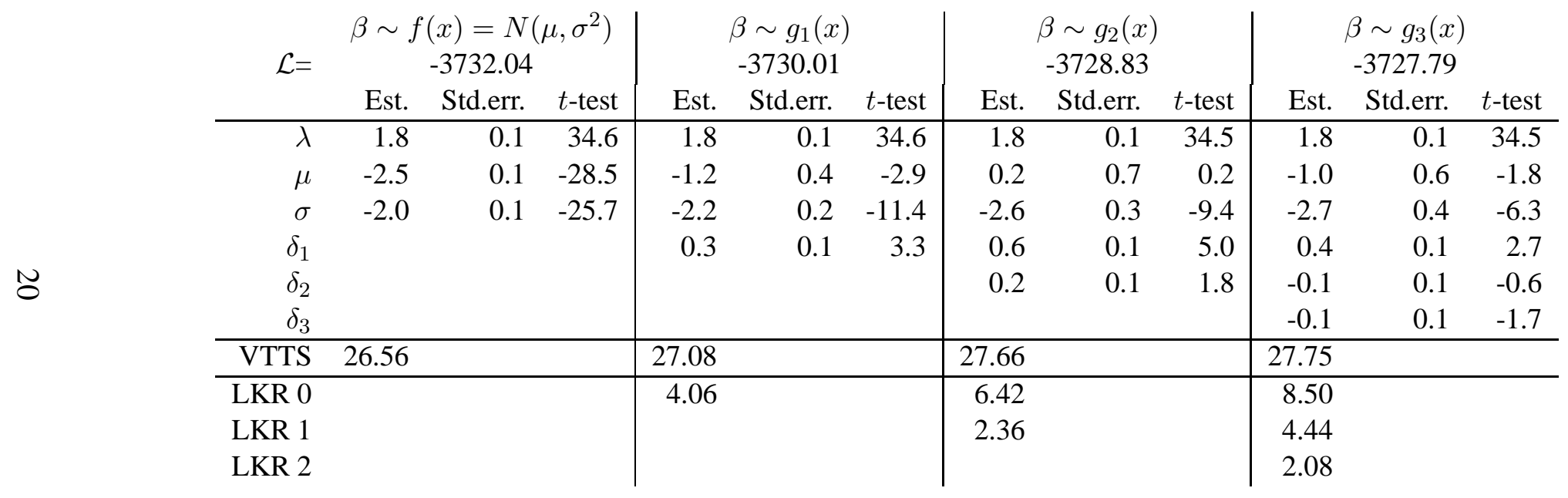

Table 6: Testing a normal distribution with more than one term 